\title{
Condición de copa de bosques y selvas de México: Análisis 2014
}

\author{
Crown condition of Mexican forests: Analysis 2014
}

\author{
Dionicio Alvarado-Rosales'; Luz de Lourdes Saavedra-Romero ${ }^{*}$, Yolanda Franco-Islas², \\ Jaime Villa-Castillo ${ }^{3}$ y David Antonio Quiroz-Reygadas ${ }^{3}$
}

\begin{abstract}
1 Colegio de Postgraduados, Campus Montecillo. Programa de Fitosanidad-Fitopatología. Montecillo, Estado de México, México.
\end{abstract}

\author{
2 Universidad Autónoma Chapingo. División de 3 Consultor independiente. \\ Ciencias Forestales. Chapingo, Estado de México, * Autor de correspondencia. saavedra.luz@colpos.mx
}

\section{RESUMEN}

Se presenta el análisis de los datos colectados en 2014 por el Inventario Nacional Forestal y de Suelos (INFyS), para el indicador de salud forestal denominado condición de copa, el cual incluyó 88162 árboles localizados en 1443 conglomerados. Las variables analizadas fueron: proporción de copa viva (Pcv), densidad de copa (DenC), transparencia de follaje (TraF) y muerte regresiva (Mreg), para las que se obtuvieron estadísticos descriptivos por géneros combinados, por grupo (coníferas y latifoliadas) y por género. También, se categorizó su salud con base en umbrales propuestos en estudios previos. Los valores promedio de Pcv, DenC, TraF, Mreg para géneros combinados fueron $35.9 \%, 44.4 \%, 50.6 \%$ y $2.6 \%$, respectivamente, mientras que para latifoliadas fueron de forma recíproca $32.7 \%$, 44.2\%, 50.5\% y $2.7 \%$ y, en coníferas, fueron $47.5 \%, 46.2 \%, 51.1 \%$ y $1.2 \%$. Se presentan promedios para 18 géneros con n $\geq 1000$. En cuanto a la salud, más de 50\% de los árboles presentó una Pcv en la categoría pobre, 27.9\% una DenC en la misma categoría, 60.26\% una TraF severa y menos de 1\%, una Mreg severa. Cincuenta y cuatro punto dos de cada cien de las latifoliadas presentaron Pcv pobre y $67 \%$ de las coníferas una TraF severa. Nayarit y Jalisco presentaron el arbolado de menor calidad de copas. Es prioritario generar estándares de copa propios, capacitar a las brigadas de campo y realizar las mediciones en la época apropiada. Este es el primer estudio sobre el indicador condición de copa en bosques y selvas de México, producto del esfuerzo del INFyS y la Gerencia de Sanidad de la Comisión Nacional Forestal.

PALABRAS CLAVE: categorías de salud; densidad de copa; indicador de salud forestal; muerte regresiva; transparencia de follaje.

\section{ABSTRACT}

Data analysis of the health indicator crown condition is presented for the data collected in 2014 by the National Forest and Soils Inventory (NFSI); it included 88162 trees localized in 1443 plots. The analyzed variables were: Live crown ratio (Lcr), Crown density (Cden), Foliage transparency (Ftra), and Crown dieback (Cdie). Descriptive statistics were obtained for each one, for all the trees, by group (conifers and hardwoods), and by genus. Tree health was categorized based on thresholds proposed in previous studies. The average values for Lcr, Cden, Ftra, Cdie for all the trees were $35.9 \%, 44.4 \%, 50.6 \%$ and $2.6 \%$, respectively, meanwhile for hardwoods $32.7 \%$, $44.2 \%, 50.5 \%$ and $2.7 \%$ were the reciprocal, and in conifers they were $47.5 \%, 46.2 \%, 51.1 \%$ and $1.2 \%$. Averages for 18 genera with $\mathrm{n} \geq$ 1000 were obtained. Regarding health, more than 50\% of the trees showed Lcr in the poor category, $27.9 \%$ in the same category for Cden, $60.26 \%$ with severe Ftra and less than 1\%, severe Cdie. $54.2 \%$ of the hardwoods showed poor Lcr, and $67 \%$ of the conifers severe Ftra. Nayarit and Jalisco states presented the lowest quality of tree crowns. It is a priority to generate our tree crown standards, to train field crews, and perform measurements in the right season. This is the first analysis of crown condition health indicator for Mexican forests, a joint effort of the NFSI and the Forest Health Department of the National Forestry Commission.

KEYWORDS: health categories; crown density; forest health indicators; crown dieback; foliage transparency. 


\section{INTRODUCCIÓN}

Los países miembros del protocolo de Montreal, entre los que se encuentra México, contienen más de $90 \%$ de los bosques templados y boreales del mundo (The Montreal Process, 2015). Tomando como referencia el compromiso que adquirió cada nación respecto a evaluar y conocer la condición de su recurso forestal (United States Department of Agriculture [USDA], 2004; Ochoa, 2001), en México, el Inventario Nacional Forestal y de Suelos (INFyS) y la Gerencia de Sanidad de la Comisión Nacional Forestal (Conafor) acordaron iniciar en 2012 la medición de dos indicadores de salud forestal, denominados "condición de copa" y "daños al arbolado", con el objetivo de determinar el estado de salud actual de los bosques y selvas de México.

En las últimas décadas, se han desarrollado métodos integrales para evaluar y monitorear la salud de los bosques templados, boreales y subtropicales del mundo, así como para la detección temprana de problemas fitosanitarios (Michel y Seidling, 2017; Pontius y Hallet, 2014; Brandeis y Turner, 2013). En este contexto, uno de los enfoques de mayor uso hoy día son las evaluaciones de copa, ya que, a través de ellas, es posible distinguir los primeros síntomas de estrés en los árboles, los cuales se pueden manifestar no solo a través de la cantidad de follaje presente, sino también a través de su distribución a lo largo del tronco (Tkacz, Moody, Villa-Castillo y Fenn, 2008; Zarnoch, Bechtold y Stolte, 2004).

La evaluación de la salud forestal a gran escala fue iniciada en 1990 en Estados Unidos de América por el Programa de Monitoreo de Salud Forestal (FHMP) del Servicio Forestal de ese país (Randolph y Thompson, 2010; Riitters y Tkacz, 2004), desde entonces, la condición de copa como indicador de la salud del bosque y del árbol ha mostrado su eficiencia en la identificación de síntomas de estrés y declinación temprana, hasta la caracterización de síntomas que reflejan su inminente mortalidad (Pontius y Hallett, 2014; Morin, Steinman y Randolph, 2012). La pérdida de follaje y ramas y sus patrones de distribución no solo alteran la apariencia y forma de las copas, sino que afectan también la tasa fotosintética y la productividad. Debido a esto, los árboles densamente foliados suelen asociarse con tasas altas de crecimiento, mientras que las copas pequeñas y con follaje disperso se asocian a un estado de declinación (Schomaker et al., 2007). Típicamente, la degradación de la copa es resultado de la incidencia de factores de estrés pasados y presentes y, en general, su apariencia puede proveer una medida integrada de las condiciones del sitio, densidad del rodal e influencia externa de distintos agentes (enfermedades, insectos, eventos climáticos, senescencia, etc.), los que, cuando son suficientemente severos, pueden desencadenar en la muerte del árbol (Lawrence, Moltzan y Moser, 2002; Kenk, 1993). Entre las mediciones de copa de mayor uso destacan la eficiencia fotosintética, la proporción de copa viva, la transparencia de follaje, densidad de copa y muerte regresiva (Pontius y Hallet, 2014; Saavedra-Romero et al., 2016).

En este sentido, la meta de la Gerencia de Sanidad y del INFyS es continuar con la medición anual y el análisis de las diferentes variables de copa en la red de sitios que se tienen en el país. Además, con los resultados del presente análisis, se busca proveer de información sobre el estado de salud actual de las selvas y bosques mexicanos. Finalmente, se espera, que en el corto plazo se incluyan otros indicadores de salud, como los que actualmente mide el FIA de Estados Unidos, sin embargo, cabe reconocer que este es un paso importante que la Gerencia de Sanidad y el INFyS de la CONAFOR han dado. Es por ello, que por vez primera para el país, se plantearon los objetivos del presente análisis.

\section{OBJETIVOS}

1) Obtener un resumen de los atributos estadísticos de las variables absolutas de copa a partir de la base de datos del INFyS 2014 y 2) generar categorías de salud poblacionales, por grupo y para los géneros arbóreos de mayor frecuencia en las selvas y bosques mexicanos. 


\section{MATERIALES Y MÉTODOS}

\section{Base de datos}

Los datos analizados en el presente trabajo fueron obtenidos en las mediciones realizadas por el Inventario Nacional Forestal y de Suelos 2014, para el indicador condición de copa en 1443 conglomerados.

\section{Diseño de muestreo}

El diseño utilizado por el INFyS para el levantamiento de datos fue el muestreo sistemático estratificado por conglomerados. Para ello, las áreas boscosas y selváticas del país se dividieron en paneles cuadrangulares $(5 \mathrm{~km} \times 5 \mathrm{~km})$, estrategia que permitió obtener una distribución espacial y regular. La unidad primaria de muestreo o conglomerado estuvo integrada por cuatro sitios de $400 \mathrm{~m}^{2}(\mathrm{r}=11.28 \mathrm{~m})$, en un arreglo de " $Y$ " invertida. Para bosques templados, los sitios fueron circulares; en el caso de selvas cálido-secas y cálido-húmedas, los sitios fueron rectangulares (Inventario Nacional Forestal y de Suelos [INFyS], 2012).

\section{Medición del indicador condición de copa}

La evaluación de copa se aplicó al estrato arbóreo con diámetro normal $\geq 7.5 \mathrm{~cm}$ medido a $1.3 \mathrm{~m}$ sobre el nivel del suelo y en árboles con copa definida (Schomaker et al., 2007; United States Department of Agriculture [USDA], 2005). Se evaluaron visualmente cuatro variables absolutas a través de las cuales se estimó la cantidad, condición y distribución de la biomasa presente en la copa. La evaluación fue realizada por brigadas de dos personas, previamente capacitadas. Las variables de copa incluidas en este análisis fueron: (1) proporción de copa viva (Pcv, \%) - relación entre el largo de copa y la altura total del árbol; (2) densidad de copa (DenC, \%) — cantidad de ramas, follaje y estructuras reproductivas que bloquean la luz que pasa a través de la copa; (3) transparencia de follaje (TraF, $\%$ - cantidad de luz que pasa a través de la porción viva y normalmente foliada de la copa; y (4) muerte regresiva (Mreg, \%) — mortalidad reciente de ramas con ramillas finas, la cual inicia en la parte alta y terminal del árbol y progresa hacia abajo, estas variables se registraron en intervalos de 5\% (Schomaker et al., 2007).

\section{Análisis de datos}

Previo al análisis estadístico, se realizó la depuración de la base de datos con el propósito de asegurar la calidad especificada en los protocolos de evaluación del indicador condición de copa (Schomaker et al., 2007; USDA, 2005). Posteriormente, se obtuvieron histogramas de frecuencia y estadísticos descriptivos generales $(\mathrm{N}$, media, error estándar, intervalos de confianza, valor mínimo, máximo y mediana), para las cuatro variables absolutas de copa ( $\mathrm{Pcv}$, DenC, TraF y Mreg). El análisis se realizó de forma combinada, considerando todos los géneros arbóreos por grupo (latifoliadas y coníferas) y por género individual. Los árboles con follaje verde pero que no presentaron una copa definida, por ejemplo: 0\% de DenC y Pcv; 95\% de Mreg y 99\% de TraF se omitieron del análisis, así como aquellos con alturas menores a $2 \mathrm{~m}$ y diámetro normal menor de 7.5 cm (Randolph, 2004). El análisis se realizó con el software $\mathrm{SAS}^{\circledR} 9.0$.

\section{Categorías de salud}

Con base en los umbrales inicialmente establecidos por Bechtold, Hoffard y Anderson (1992) y modificados por Randolph (2004) y Randolph y Moser (2004), se categorizó la condición de salud del arbolado (Tabla 1). Cabe mencionar que, ante la falta de umbrales para Pcv, se tomó como referente el porcentaje establecido por Widmann $e t$ al., (2012; 20\%) para calificar copas con pobre vigor; sin embargo, en el presente trabajo, se consideró 30\% para la categoría pobre, con el fin de no subestimar la condición de los árboles. 
TABLA 1. Categorías de salud para las variables absolutas del indicador condición de copa (Randolph, 2004; Randolph y Moser, 2004; Bechtold et al., 1992).

\begin{tabular}{cccc}
\hline $\begin{array}{c}\text { Proporción de copa } \\
\text { viva (Pcv) }\end{array}$ & $\begin{array}{c}\text { Densidad de copa } \\
\text { (DenC) }\end{array}$ & $\begin{array}{c}\text { Transparencia de follaje } \\
\text { (TraF) }\end{array}$ & $\begin{array}{c}\text { Muerte regresiva } \\
\text { (Mreg) }\end{array}$ \\
\hline Buena $>50 \%$ & Óptima $51 \%-100 \%$ & Normal $<20 \%$ & Ninguna $\leq 5 \%$ \\
Moderada 31\%-50\%. & Buena 41\%-50\% & Moderada 21\%-40\% & Ligera 6\%-20\% \\
Pobre $<30 \%$ & Moderada 31\%-40\% & Severa 41\%-100\% & Moderada 21\%-35\% \\
& Pobre 0\%-30\% & & Severa 36\%-100\% \\
\hline
\end{tabular}

\section{RESULTADOS}

\section{Condición de copa}

\section{Por géneros combinados}

El número total de árboles evaluados por el INFyS en 2014 fue de 88 162, en 1443 conglomerados y para 30 estados de México. El número de registros para bosques fue 29934 árboles y 58228 para selvas. La riqueza de especies fue 979 y la riqueza de géneros fue 360 , siendo los más frecuentes Quercus, Pinus y Bursera.

Los histogramas de frecuencia y frecuencia acumulada de las cuatro variables absolutas de copa de todos los géneros arbóreos combinados se muestran en la figura 1. El promedio porcentual de cada una fue: $33.85-\mathrm{Pcv}, 44.4-$ DenC, 50.56-TraF y 2.62-Mreg (Tabla 2). A diferencia de la Mreg, el resto de las variables presentaron una distribución amplia.

\section{Por grupo}

Se observaron enormes variaciones en el análisis por grupos; las coníferas incluyeron $7.44 \%$ de los registros y las latifoliadas $92.55 \%$. En las primeras, los porcentajes promedio de copa, fueron 47.52-Pcv, 46.27-DenC, 51.19TraF y 1.24 de Mreg; respecto al segundo grupo, los valores fueron ligeramente menores, especialmente en la Pcv, con $32.75 \%$, 44.29\%-DenC, 50.51\%-TraF y sutilmente más alta la Mreg, con $2.73 \%$ (Tabla 2).

\section{Por género arbóreo}

El porcentaje promedio de las variables de copa para 18 géneros con más de mil registros ( $\mathrm{n}>1000$ ), se presentan en las tablas 3, 4, 5 y 6. La Pcv osciló del valor más bajo con $19.65 \%$ para Gymnopodium sp., hasta $51.22 \%$ en Quercus sp. (Tabla 3). La DenC fluctuó entre 38.77\% para el género Haematoxylum sp. y 49.95\% en Manilkara sp. (Tabla 4). Respecto a la TraF, el menor valor fue $44.71 \%$ en Pouteria sp. y el más alto $55.89 \%$ en Lysiloma sp. (Tabla 5). Finalmente, la Mreg más baja fue $0.33 \%$ en Diospyros sp. y $8.6 \%$ para Lysiloma sp. (Tabla 6). También se incluyen los intervalos de confianza a 95\% de cada variable. Cabe mencionar que, debido a la importancia ecológica que tienen para México, destacan por su frecuencia, los géneros Pinus y Quercus.

\section{Por entidad federativa}

Los valores promedio ( \pm error estándar) de las variables de copa por estado se incluyen en la figura 2A-C, destacando por sus bajas Pcv los árboles evaluados en Quintana Roo con $20.84 \%$ y por las más altas, Aguascalientes (60.61\%). La DenC fue mayor para los árboles de Tamaulipas con $67.76 \%$ y menor en Nayarit con $22.08 \%$. Las mayores TraF se registraron en Hidalgo con $74.77 \%$ y en Aguascalientes con $21.36 \%$. Respecto a la muerte regresiva, Nayarit presentó $36.61 \% \quad$ y $\quad$ se observó insustancial para Aguascalientes. 

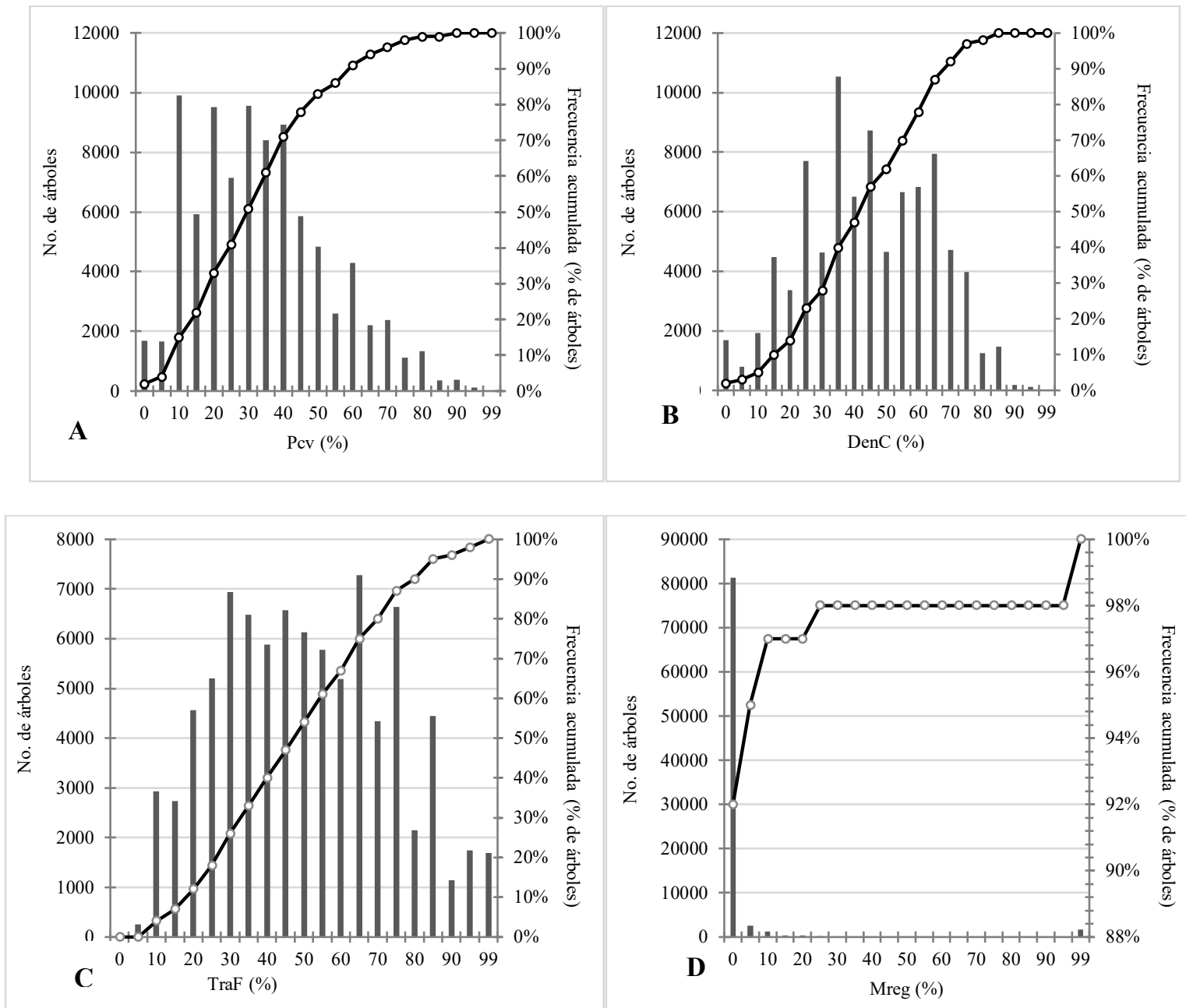

FIGURA 1. Histogramas de frecuencia y distribución de frecuencia acumulada para Pcv (A), DenC (B), TraF (C) y Mreg (D), para todos los géneros combinados (Datos INFyS, 2014).

TABLA 2. Atributos medios de copa y otros estadísticos para árboles de latifoliadas, coníferas y total de árboles con diámetro normal $\geq$ $7.5 \mathrm{~cm}$ (Datos INFyS, 2014).

\begin{tabular}{|c|c|c|c|c|c|c|c|c|c|}
\hline \multirow[b]{2}{*}{ Variable } & & \multirow[b]{2}{*}{$\mathrm{n}$} & \multicolumn{7}{|c|}{ Intervalo de confianza ${ }^{\dagger}(\%)$} \\
\hline & & & Media & E.E. $\ddagger$ & L.I. & L.S. & Min. & Med. & Máx \\
\hline \multirow{3}{*}{ PcV } & Latifoliadas & 81597 & 32.75 & 0.07 & 32.62 & 32.88 & 0 & 30 & 99 \\
\hline & Coníferas & 6565 & 47.52 & 0.20 & 47.13 & 47.92 & 0 & 45 & 99 \\
\hline & Todos los árboles & 88162 & 33.85 & 0.06 & 33.72 & 33.98 & 0 & 30 & 99 \\
\hline \multirow{3}{*}{ DenC } & Latifoliadas & 81597 & 44.29 & 0.07 & 44.15 & 44.43 & 0 & 45 & 95 \\
\hline & Coníferas & 6565 & 46.27 & 0.21 & 45.87 & 46.68 & 0 & 45 & 95 \\
\hline & Todos los árboles & 88162 & 44.40 & 0.07 & 44.31 & 44.57 & 0 & 45 & 95 \\
\hline \multirow{3}{*}{ TraF } & Latifoliadas & 81597 & 50.51 & 0.08 & 50.35 & 50.66 & 0 & 50 & 99 \\
\hline & Coníferas & 6565 & 51.19 & 0.24 & 50.72 & 51.65 & 0 & 50 & 99 \\
\hline & Todos los árboles & 88162 & 50.6 & 0.08 & 50.41 & 50.71 & 0 & 50 & 99 \\
\hline \multirow{3}{*}{ Mreg } & Latifoliadas & 81597 & 2.73 & 0.05 & 2.63 & 2.83 & 0 & 0 & 99 \\
\hline & Coníferas & 6565 & 1.24 & 0.10 & 1.04 & 1.44 & 0 & 0 & 99 \\
\hline & Todos los árboles & 88162 & 2.62 & 0.05 & 2.53 & 2.71 & 0 & 0 & 99 \\
\hline
\end{tabular}

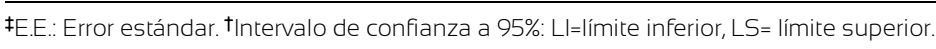


TABLA 3. Atributos medios de proporción de copa viva por género arbóreo bajo el criterio de n $\geq 1000$ (Datos INFyS, 2014).

\begin{tabular}{|c|c|c|c|c|c|c|c|c|}
\hline \multirow[b]{2}{*}{ Género } & \multirow[b]{2}{*}{$\mathrm{n}$} & \multirow[b]{2}{*}{ Media } & \multirow[b]{2}{*}{ E.E. ${ }^{\ddagger}$} & \multicolumn{2}{|c|}{ Intervalo de confianza $(95 \%)^{\dagger}$} & \multirow[b]{2}{*}{ Mín } & \multirow[b]{2}{*}{ Med. } & \multirow[b]{2}{*}{ Máx } \\
\hline & & & & L.I. & L.S. & & & \\
\hline & & 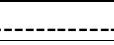 & 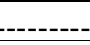 & ---------Pol & - & 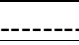 & 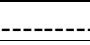 & \\
\hline Acacia & 1167 & 33.83 & 0.60 & 32.65 & 35.01 & $\mathrm{O}$ & 35 & 100 \\
\hline Brosimum & 1552 & 29.02 & 0.35 & 28.33 & 29.71 & $\mathrm{O}$ & 30 & 85 \\
\hline Bursera & 3757 & 27.20 & 0.28 & 26.65 & 27.74 & $\mathrm{O}$ & 25 & 95 \\
\hline Coccoloba & 2226 & 20.98 & 0.25 & 20.49 & 21.48 & $\mathrm{O}$ & 20 & 95 \\
\hline Croton & 1062 & 23.32 & 0.41 & 22.51 & 24.13 & 0 & 25 & 90 \\
\hline Diospyros & 1031 & 24.24 & 0.42 & 23.42 & 25.07 & $\mathrm{O}$ & 25 & 90 \\
\hline Gymnanthes & 1576 & 20.43 & 0.28 & 19.88 & 20.98 & $\mathrm{O}$ & 20 & 75 \\
\hline Gymnopodium & 2041 & 19.65 & 0.22 & 19.21 & 20.09 & $\mathrm{O}$ & 20 & 75 \\
\hline Haematoxylum & 1250 & 26.20 & 0.45 & 25.31 & 27.10 & 0 & 25 & 100 \\
\hline Heliocarpus & 1008 & 35.58 & 0.52 & 34.56 & 36.60 & $\mathrm{O}$ & 35 & 85 \\
\hline Lonchocarpus & 2674 & 22.92 & 0.22 & 22.48 & 23.36 & $\mathrm{O}$ & 20 & 80 \\
\hline Lysiloma & 2705 & 32.97 & 0.39 & 32.21 & 33.73 & $\mathrm{O}$ & 30 & 100 \\
\hline Manilkara & 2211 & 28.88 & 0.26 & 28.36 & 29.39 & 0 & 30 & 90 \\
\hline Metopium & 1822 & 24.52 & 0.29 & 23.95 & 25.09 & $\mathrm{O}$ & 20 & 90 \\
\hline Pinus & 5790 & 46.18 & 0.20 & 45.78 & 46.58 & $\mathrm{O}$ & 45 & 100 \\
\hline Pouteria & 1781 & 27.16 & 0.30 & 26.58 & 27.74 & $\mathrm{O}$ & 25 & 90 \\
\hline Quercus & 15507 & 51.22 & 0.15 & 50.93 & 51.51 & 0 & 50 & 100 \\
\hline Thouinia & 1626 & 20.19 & 0.25 & 19.70 & 20.68 & 0 & 20 & 70 \\
\hline
\end{tabular}

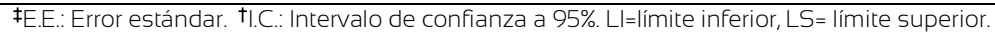

TABLA 4. Atributos medios para Densidad de copa por género arbóreo bajo el criterio de n $\geq 1000$ (Datos INFyS, 2014).

\begin{tabular}{|c|c|c|c|c|c|c|c|c|}
\hline \multirow[b]{2}{*}{ Género } & \multirow[b]{2}{*}{$\mathrm{n}$} & \multirow[b]{2}{*}{ Media } & \multirow[b]{2}{*}{ E.E. ${ }^{\ddagger}$} & \multicolumn{2}{|c|}{ Intervalo de confianza $(95 \%)^{\dagger}$} & \multirow[b]{2}{*}{ Mín } & \multirow[b]{2}{*}{ Med. } & \multirow[b]{2}{*}{ Máx } \\
\hline & & & & L.I. & L.S. & & & \\
\hline & & -------- & - & ------Po & - & 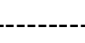 & 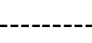 & \\
\hline Acacia & 1167 & 40.01 & 0.57 & 38.88 & 41.13 & 0 & 40 & 95 \\
\hline Brosimum & 1552 & 45.16 & 0.46 & 44.27 & 46.06 & 0 & 45 & 95 \\
\hline Bursera & 3757 & 41.82 & 0.35 & 41.13 & 42.52 & 0 & 40 & 90 \\
\hline Coccoloba & 2226 & 43.14 & 0.43 & 42.29 & 43.99 & 0 & 40 & 95 \\
\hline Croton & 1062 & 39.51 & 0.58 & 38.37 & 40.65 & 0 & 35 & 90 \\
\hline Diospyros & 1031 & 44.80 & 0.59 & 43.63 & 45.96 & 0 & 45 & 90 \\
\hline Gymnanthes & 1576 & 42.44 & 0.50 & 41.47 & 43.41 & 0 & 40 & 90 \\
\hline Gymnopodium & 2041 & 43.05 & 0.46 & 42.15 & 43.96 & 0 & 40 & 85 \\
\hline Haematoxylum & 1250 & 38.77 & 0.54 & 37.70 & 39.84 & 0 & 35 & 85 \\
\hline Heliocarpus & 1008 & 40.17 & 0.72 & 38.75 & 41.59 & 0 & 42.5 & 95 \\
\hline Lonchocarpus & 2674 & 45.45 & 0.40 & 44.67 & 46.23 & 0 & 45 & 90 \\
\hline Lysiloma & 2705 & 40.94 & 0.42 & 40.12 & 41.76 & 0 & 40 & 90 \\
\hline Manilkara & 2211 & 49.95 & 0.36 & 49.25 & 50.65 & 0 & 50 & 90 \\
\hline Metopium & 1822 & 44.80 & 0.40 & 44.01 & 45.58 & 0 & 45 & 85 \\
\hline Pinus & 5790 & 45.47 & 0.22 & 45.05 & 45.90 & 0 & 45 & 95 \\
\hline Pouteria & 1781 & 47.82 & 0.44 & 46.96 & 48.68 & 0 & 45 & 95 \\
\hline Quercus & 15507 & 48.22 & 0.16 & 47.91 & 48.53 & 0 & 50 & 95 \\
\hline Thouinia & 1626 & 43.22 & 0.49 & 42.27 & 44.18 & 0 & 40 & 85 \\
\hline
\end{tabular}

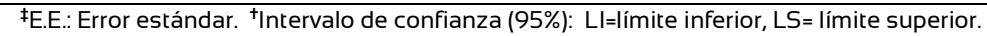


TABLA 5. Atributos medios de transparencia de follaje por género arbóreo bajo el criterio de n $\geq 1000$ (Datos INFyS, 2014).

\begin{tabular}{|c|c|c|c|c|c|c|c|c|}
\hline \multirow[b]{2}{*}{ Género } & \multirow[b]{2}{*}{$\mathrm{n}$} & \multirow[b]{2}{*}{ Media } & \multicolumn{3}{|c|}{ Intervalo de confianza $(95 \%)^{\dagger}$} & \multirow[b]{2}{*}{ Mín } & \multirow[b]{2}{*}{ Med. } & \multirow[b]{2}{*}{ Máx } \\
\hline & & & E.E. $\ddagger$ & L.I. & L.S. & & & \\
\hline & & 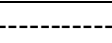 & $\cdots$ & ----Po & 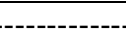 & 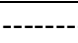 & 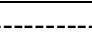 & \\
\hline Acacia & 1167 & 52.38 & 0.71 & 50.99 & 53.78 & 5 & 50 & 99 \\
\hline Brosimum & 1552 & 47.39 & 0.48 & 46.45 & 48.34 & 10 & 45 & 99 \\
\hline Bursera & 3757 & 53.81 & 0.39 & 53.05 & 54.58 & 0 & 55 & 99 \\
\hline Coccoloba & 2226 & 53.01 & 0.52 & 51.99 & 54.03 & 0 & 55 & 99 \\
\hline Croton & 1062 & 54.96 & 0.70 & 53.59 & 56.32 & 5 & 55 & 99 \\
\hline Diospyros & 1031 & 51.35 & 0.71 & 49.96 & 52.73 & 5 & 50 & 99 \\
\hline Gymnanthes & 1576 & 52.28 & 0.58 & 51.15 & 53.41 & 5 & 50 & 99 \\
\hline Gymnopodium & 2041 & 48.20 & 0.47 & 47.27 & 49.12 & 0 & 45 & 99 \\
\hline Haematoxylum & 1250 & 56.99 & 0.64 & 55.74 & 58.25 & 10 & 60 & 99 \\
\hline Heliocarpus & 1008 & 55.13 & 0.81 & 53.53 & 56.72 & 5 & 55 & 99 \\
\hline Lonchocarpus & 2674 & 46.28 & 0.46 & 45.38 & 47.19 & 0 & 45 & 99 \\
\hline Lysiloma & 2705 & 55.89 & 0.46 & 54.98 & 56.80 & 0 & 55 & 99 \\
\hline Manilkara & 2211 & 45.50 & 0.41 & 44.70 & 46.30 & 0 & 45 & 99 \\
\hline Metopium & 1822 & 50.22 & 0.46 & 49.32 & 51.13 & 0 & 50 & 99 \\
\hline Pinus & 5790 & 52.28 & 0.25 & 51.79 & 52.77 & 0 & 50 & 99 \\
\hline Pouteria & 1781 & 44.71 & 0.49 & 43.75 & 45.68 & 5 & 45 & 99 \\
\hline Quercus & 15507 & 49.51 & 0.17 & 49.17 & 49.85 & 0 & 50 & 99 \\
\hline Thouinia & 1626 & 49.43 & 0.53 & 48.39 & 50.47 & 0 & 50 & 99 \\
\hline
\end{tabular}

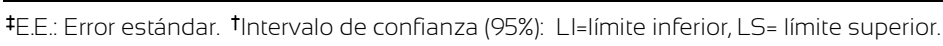

TABLA 6. Atributos medios de muerte regresiva por género arbóreo bajo el criterio de $\mathrm{n} \geq 1000$ (Datos INFyS, 2014).

\begin{tabular}{|c|c|c|c|c|c|c|c|c|}
\hline \multirow[b]{2}{*}{ Género } & \multirow[b]{2}{*}{$\mathrm{n}$} & \multirow[b]{2}{*}{ Media } & \multirow[b]{2}{*}{ E.E. ${ }^{\prime}$} & \multicolumn{2}{|c|}{ Intervalo de confianza $(95 \%)^{\dagger}$} & \multirow[b]{2}{*}{ Mín } & \multirow[b]{2}{*}{ Med. } & \multirow[b]{2}{*}{ Máx } \\
\hline & & & & L.I. & L.S. & & & \\
\hline & & 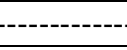 & $-\cdots$ & --------Po & - n & 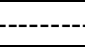 & 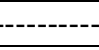 & \\
\hline Acacia & 1167 & 3.38 & 0.48 & 2.43 & 4.33 & 0 & 0 & 99 \\
\hline Brosimum & 1552 & 1.85 & 0.33 & 1.19 & 2.51 & 0 & 0 & 99 \\
\hline Bursera & 3757 & 3.25 & 0.27 & 2.73 & 3.78 & 0 & 0 & 99 \\
\hline Coccoloba & 2226 & 1.42 & 0.22 & 0.99 & 1.84 & 0 & 0 & 99 \\
\hline Croton & 1062 & 1.15 & 0.30 & 0.57 & 1.74 & 0 & 0 & 99 \\
\hline Diospyros & 1031 & 0.33 & 0.14 & 0.04 & 0.61 & 0 & 0 & 99 \\
\hline Gymnanthes & 1576 & 1.58 & 0.29 & 1.00 & 2.16 & 0 & 0 & 99 \\
\hline Gymnopodium & 2041 & 0.58 & 0.15 & 0.29 & 0.88 & 0 & 0 & 99 \\
\hline Haematoxylum & 1250 & 2.69 & 0.30 & 2.10 & 3.28 & 0 & 0 & 99 \\
\hline Heliocarpus & 1008 & 7.21 & 0.79 & 5.67 & 8.76 & 0 & 0 & 99 \\
\hline Lonchocarpus & 2674 & 0.93 & 0.17 & 0.59 & 1.26 & 0 & 0 & 99 \\
\hline Lysiloma & 2705 & 8.60 & 0.51 & 7.60 & 9.60 & 0 & 0 & 99 \\
\hline Manilkara & 2211 & 0.77 & 0.17 & 0.44 & 1.10 & 0 & 0 & 99 \\
\hline Metopium & 1822 & 1.06 & 0.22 & 0.62 & 1.50 & 0 & 0 & 99 \\
\hline Pinus & 5790 & 1.03 & 0.10 & 0.84 & 1.22 & 0 & 0 & 99 \\
\hline Pouteria & 1781 & 1.15 & 0.24 & 0.67 & 1.63 & 0 & 0 & 99 \\
\hline Quercus & 15507 & 4.01 & 0.13 & 3.76 & 4.26 & 0 & 0 & 100 \\
\hline Thouinia & 1626 & 1.48 & 0.28 & 0.93 & 2.03 & 0 & 0 & 99 \\
\hline
\end{tabular}

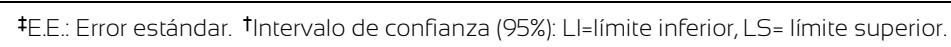




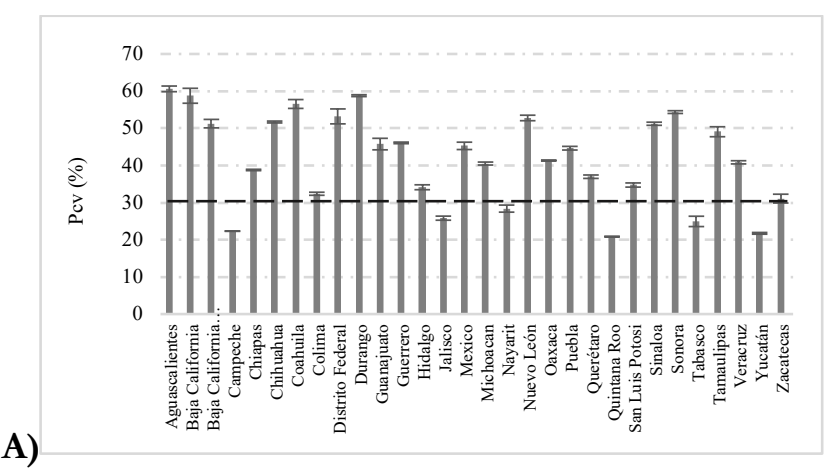

A)

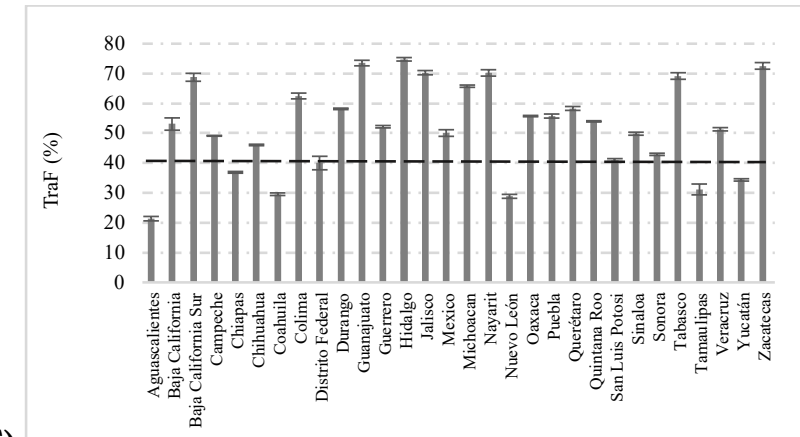

C)

Figura 2. Promedio ( \pm error estándar) de las variables absolutas de copa por entidad federativa. A: Pcv; B: DenC; C: TraF; D: Mreg. Valores por debajo de la línea punteada se califican como pobres en el caso de Pcv y Denc. Para TraF y Mreg, los que están por arriba se califican como severas de acuerdo con Randolph, (2004) y Randolph y Moser (2004)

\section{Categorías de salud}

Empleando los umbrales de salud modificados por Randolph (2004), Randolph y Moser (2004) y descritos en la sección de Métodos, se encontró que $51.5 \%$ de los géneros arbóreos combinados presentó una Pcv en la categoría pobre, lo mismo para DenC en $27.85 \%$ de los árboles; $60.26 \%$ una TraF severa y una Mreg calificada como insignificante (Fig. 3A-C). Las latifoliadas destacaron por su Pcv pobre en $54.2 \%$ de los árboles, $28.61 \%$ con una pobre DenC y $60 \%$ con TraF severa, siendo esta, más alta en coníferas. En este grupo, solo 16.45\% de los árboles presentó una Pcv pobre; en el caso de la DenC, 18.56\% de las copas se categorizaron en condición pobre. En general, los porcentajes de muerte regresiva fueron muy bajos (Fig. 3). Respecto a los géneros individuales más frecuentes, Pinus y Quercus y Bursera y Lysiloma, todos mostraron una TraF en la categoría severa. Bursera también presentó una pobre Pcv y Lysiloma una ligera Mreg.
B)
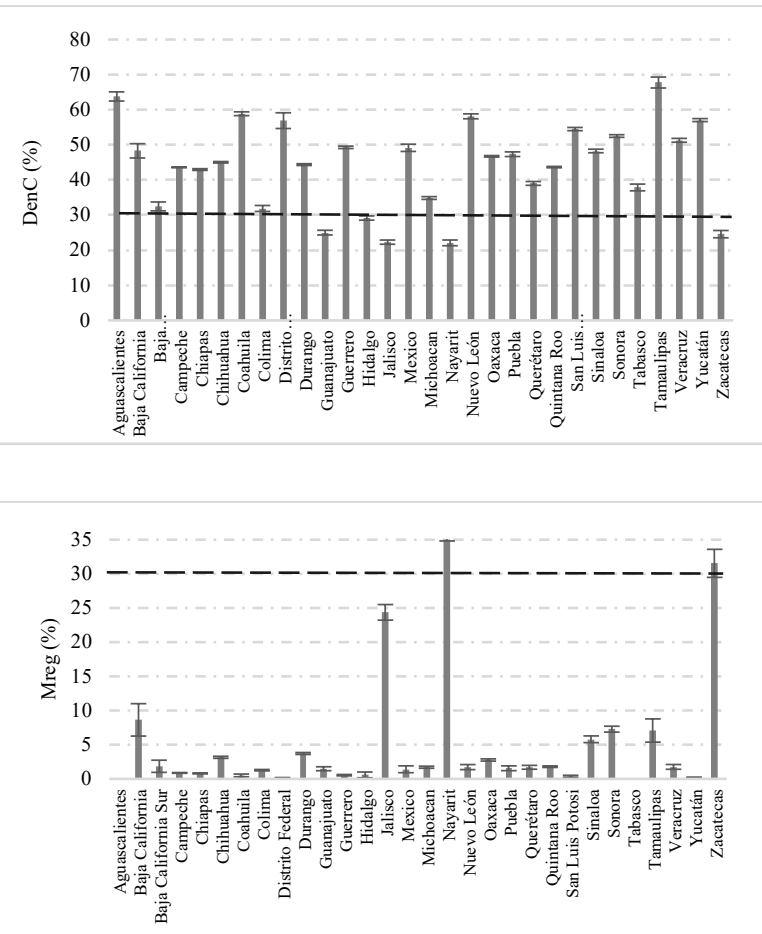

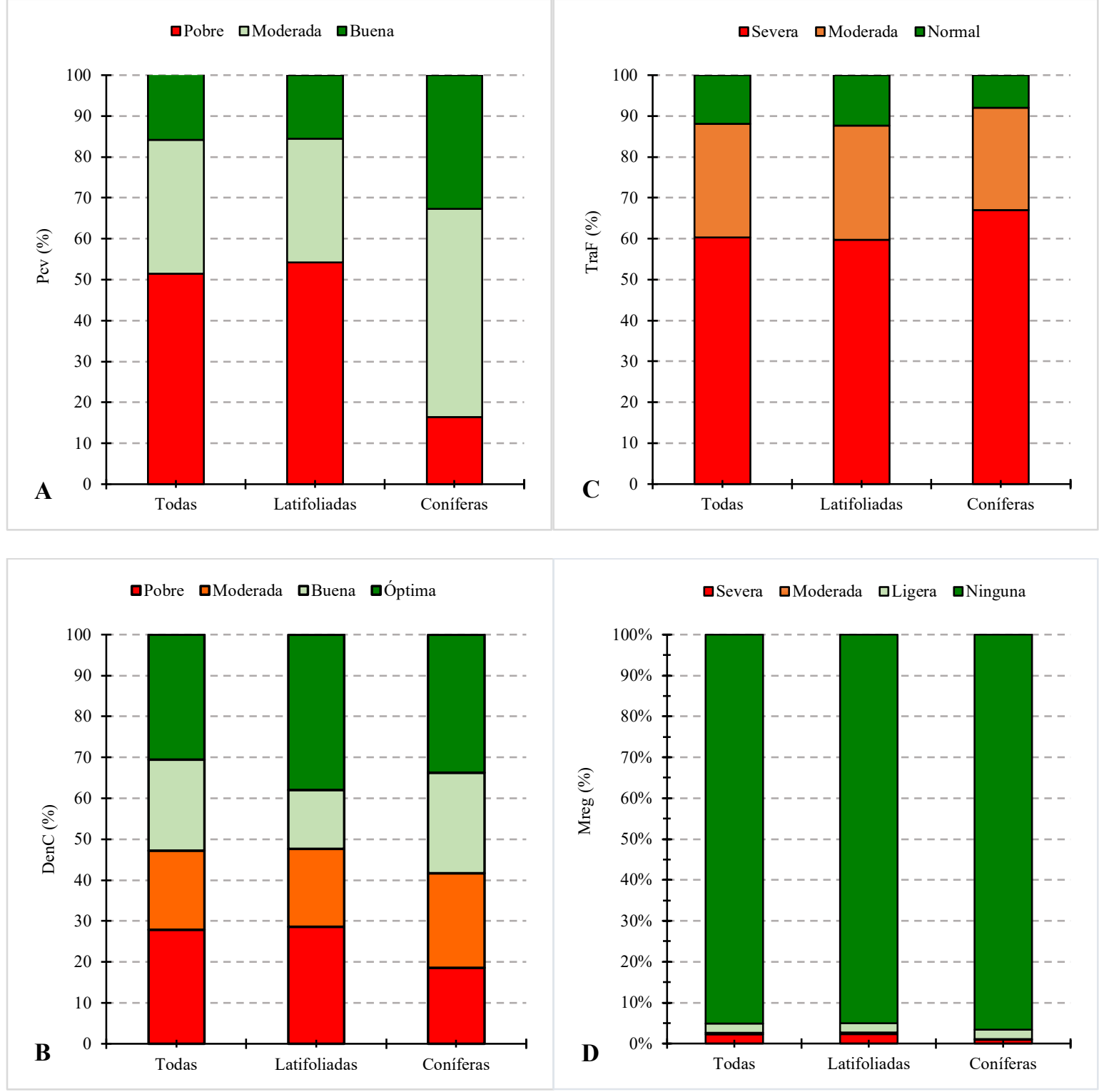

FIgURA 3. Categoría de salud para todos los árboles y por grupo (latifoliadas y coníferas). A: Proporción de copa viva (Pcv); B: Densidad de copa (DenC); C: Transparencia de follaje (TraF) y D: Muerte regresiva (Mreg) Datos INFyS (2014).

\section{DISCUSIÓN}

Géneros combinados, individuales y por entidad federativa

Los valores promedio de Pcv, DenC, TraF y Mreg, por géneros combinados $(33.85 \%, 44.4 \%, 50.6 \%$ y $2.62 \%$, respectivamente), obtenidos en el presente estudio, presentaron variabilidad, similar a lo señalado en otras investigaciones. Cabe mencionar que, ante la carencia de publicaciones científicas sobre el indicador condición de copa de bosques naturales en México, se tomaron como referente los resultados obtenidos por Randolph, Morin y Steinman (2010a; 2010b), Randolph, Campbell y Christensen (2010) y Randolph y Thompson (2010), para especies arbóreas de Estados Unidos de América. Con base en estos, se observó que la DenC de sus especies 
combinadas fue sutilmente mayor, con $46.5 \%$, mientras que la $\operatorname{TraF}$ con $18.4 \%$ es, por mucho, menor respecto a lo obtenido en el presente trabajo. La Mreg, por su parte, fue menor para los árboles evaluados en México. En cuanto a la Pcv, Pontius y Hallet (2014) observaron valores promedio que oscilan entre $46.17 \%$ y $59.79 \%$, para cuatro latifoliadas y una conífera del Noreste de Estados Unidos (Acer saccharum Marshall, Fagus grandifolia Ehrh., Fraxinus americana L., Quercus rubra L. y Tsuga canadensis L. Carriére), superior al obtenido en el presente. Por otro lado, para especies de Checoslovaquia (latifoliadas y coníferas) se señalan valores promedio de 56\% (Sharma et al., 2017), también por arriba del obtenido, mientras que para 21 latifoliadas de la cuenca del Estuario de San Juan, Puerto Rico se obtuvieron valores de $22.7 \%$ a $88.6 \%$ (Brandeis, Escobedo, Staudhammer, Nowak y Wayne, 2014).

Respecto al análisis por género individual, también se presentaron enormes variaciones (Tablas 3, 4, 5 y 6), de manera análoga a los resultados de Randolph (2006), quien realizó el análisis a nivel específico (53 en total). Por ejemplo, para Bursera, Lysiloma, Pinus y Quercus, cuatro de los géneros más frecuentes evaluados por el INFyS en 2014, sus $\mathrm{Pcv}$ fueron de $27.2 \%, 32.9 \%, 46.2 \%$ y $51.2 \%$, respectivamente. Las DenC, oscilaron entre $40 \%$ y $45 \%$, la TraF entre 50\% y 55\%, mientras que la Mreg fue mayor en Lysiloma con $8.6 \%$.

Según Westfall, Westfall y Randolph (2019), entre las variables de copa estudiadas a la fecha, la Pcv ha mostrado cualidades diversas; mediante ella, es posible tasar el potencial de crecimiento de los árboles (vigor), predecir sus probabilidades de muerte y profundizar en el estudio conductual de incendios forestales. Debido a ello, la reducción gradual del dosel se puede calificar como síntoma terminal de deterioro y como respuesta a severo estrés. La Pcv, también permite detectar cambios sutiles de síntomas de estrés en periodos cortos (Pontius y Hallet, 2014); sin embargo, pese a sus virtudes, no se le ha conferido la importancia que merece (Morin et al., 2012; Schomaker et al., 2007).

\section{Categorías de salud}

Hoy día, una de las alternativas más viables para definir y generar umbrales o categorías discretas de salud arbórea es el análisis estadístico (Schomaker et al., 2007; Bechtold et al., 1992; Randolph, 2004); desafortunadamente, existen ciertas limitantes en ello, ya que estas categorías no toman en cuenta las diferencias inter o intraespecíficas de morfología de ramas y hojas y su distribución (Bechtold y Randolph, 2018), tampoco consideran la gama de condiciones geográficas y de calidad de sitio (Oswalt et al., 2009), con excepción de los estudios de Morin, Randolph y Steinman (2015); por ello, existe el riesgo de calificar erróneamente los árboles como saludables cuando en la realidad no lo son y viceversa (Randolph, 2009; Schomaker et al., 2007). Con este enfoque y ante el hecho de que México no cuenta con umbrales propios para las variables Pcv, DenC, TraF y Mreg, se tomaron como base los estudios de Randolph (2004) y Randolph y Moser (2004) (Tabla 1) para categorizar la salud de los árboles evaluados por el INFyS en 2014, considerando, desde luego, los pros y contras mencionados anteriormente.

Con base en lo anterior, más de la mitad de los géneros arbóreos combinados presentaron una Pcv en la categoría pobre, es decir, $<30 \%$ y, en una categoría buena, $16.8 \%$. Por grupo, la categoría pobre se manifestó en $54.3 \%$ de las latifoliadas y en $16.5 \%$ de las coníferas (Fig. 3A), no obstante, se observó que estos árboles se hallaban concentrados en una región específica de del país (el sureste mexicano), representado por Campeche, Quintana Roo, Tabasco y Yucatán, además de Nayarit y Jalisco, al oeste (Fig. 2A). Respecto a géneros individuales, al menos 12 de ellos se encontraron en la categoría pobre de Pcv (Tabla 3), siendo Gymnopodium el del valor más bajo (19.65\%) y Quercus $(51.22 \%)$ el más alto (categoría de salud buena). Si bien son diversos los factores que pueden estar asociados a estos resultados, es posible que las bajas Pcr sean resultado de diferentes condiciones fisiogeográficas y climáticas, de la fenología, del tipo de bosque y fechas de medición; la posición sociológica de los árboles y la densidad también 
pudieron afectar la cantidad de biomasa presente al momento de realizar las evaluaciones, por ejemplo, árboles en rodales con baja densidad tienden a mayores porcentajes de Pcv (Hasenauer y Monserud, 1996) y, a medida que la competencia por luz aumenta, la Pcv disminuye, especialmente en individuos suprimidos e intermedios (Holdaway, 1986). Árboles de crecimiento rápido también suelen tener copas más alargadas y anchas que los árboles de crecimiento lento (Anderson y Belanger, 1986).

Respecto a la DenC y la TraF, Pontius y Hallet (2014) mencionan que toda vez que el follaje pierde su capacidad fotosintética, el árbol tiende a deshacerse del tejido foliar que no le aporta ganancia de carbohidratos; este fenómeno, que puede ser por causas naturales (p.e. senescencia), también puede ser en respuesta a un estrés temprano ocasionado por la presencia de plagas o enfermedades. En torno a estas variables, la DenC para los géneros combinados, se ubicó en la categoría pobre para 27.86\% y casi un tercio del total en la categoría de densidad óptima (Fig. 3B). Por su parte, $28.6 \%$ de las latifoliadas presentaron un categoría pobre y en coníferas, 18.6\% (Fig. 3B). A nivel estatal, Guanajuato, Jalisco y Zacatecas presentaron arbolado en la categoría pobre (Fig. 2B). La TraF fue severa en $60.36 \%$ de la población y $11.9 \%$ en categoría normal (Fig. 3C). Para latifoliadas, la categoría severa estuvo representada por $60 \%$ de los individuos y en coníferas en $66.1 \%$. Por entidad federativa, al menos 22 estados presentaron arbolado en la categoría severa de TraF (Fig. 2C).

Para bosques subtropicales y considerando la posición sociológica de los árboles, Brandeis y Turner (2013) observaron densidades de copa de $8 \%$ a $45 \%$ y transparencias foliares de $10 \%$ a $55 \%$, calificándolas como normales. De acuerdo con Morin et al. (2015), la magnitud de la TraF es de suma importancia para tipificar y predecir la salud y sobrevivencia del árbol, no obstante, un valor alto para esta variable no implica, necesariamente, un juicio negativo sobre la salud, ya que ello puede ser resultado de un crecimiento acelerado y vigoroso (Ferretti, 1998). La apertura repentina de claros dentro del bosque por la muerte de árboles dominantes o por la caída de árboles vecinos puede ocasionar mayor penetración de luz al sotobosque, afectando negativamente los niveles de transparencia en especies tolerantes, e incrementar las tasas de crecimiento en las intolerantes (Krocher y Harris, 2007).

Respecto a los resultados obtenidos para Mreg, 95\% de la población presentó un valor insignificante $(\leq 5 \%)$ y $2.24 \%$ una categoría severa (Fig. 3D). Jalisco, Nayarit y Zacatecas fueron los estados que presentaron una categoría de Mreg de moderada a severa (Fig. 2D). De acuerdo con Oak, Steinman, Starkey y Yockey (2004), el síntoma que separa la declinación del encino, de otras enfermedades, es la muerte regresiva (lenta y progresiva muerte de ramas y ramillas). Las causas de este fenómeno son complejas, pero comúnmente se asocian al cambio climático (incremento en la temperatura, sequías prolongadas, etc.), presencia de plagas y enfermedades, o la sinergia entre estos y otros factores (Martínez-Vilalta, Lloret y Breshears, 2012; McDowell et al., 2013). Por sí misma, la Mreg se manifiesta como respuesta a un estrés moderado-reciente (Pontius y Hallet, 2014) y, aunque bajos porcentajes en esta pueden no afectar al desarrollo de árboles individuales, es posible observar los primeros síntomas de deterioro en la copa cuando es > 25\% (Millers et al., 1989). Steinman (2000), por otra parte, concluye que un árbol tiene mayor probabilidad de morir en un plazo anual cuando su Mreg excede $20 \%$ en coníferas y $30 \%$ en latifoliadas, con estos límites sería posible establecer si los árboles se están acercando a un nivel crítico de estrés o a su mortalidad inminente (Randolph, 2004).

Pese a que los umbrales de salud empleados como referente fueron obtenidos para distintas condiciones fisiogeográficas y especies arbóreas, los resultados del presente análisis serán un parteaguas para futuras investigaciones. Programar adecuadamente el levantamiento de información en campo en función de las fechas de máxima expansión foliar y de la distribución natural de las especies de bosques y selvas proporcionará mayor certidumbre sobre si las magnitudes de las variables de copa son "normales". Idealmente, los umbrales deberían desarrollarse con bases biológicas para especies individuales, sin embargo, se requiere establecer 
correlaciones entre este indicador de salud y las posibles pérdidas derivadas de la mortalidad de árboles y la disminución en las tasas de crecimiento (Bechtold y Randolph, 2018). Realizar mediciones periódicas de copa proporcionará un panorama más amplio y confiable al momento de aplicar umbrales, bien sean estadísticos o biológicos. Finalmente, existen razones que hacen que la evaluación y el monitoreo de la salud forestal sean de importancia crítica (Ferretti, 1997), en primer lugar, porque los bosques son recursos ecológicos, económicos y estéticos invaluables en los que se basa la autorregulación del planeta y, en segundo lugar, como cualquier otro recurso ambiental, el manejo adecuado de los bosques debe sustentarse en el conocimiento de su estado actual.

\section{CONCLUSIONES}

La magnitud de las variables del indicador condición de copa obtenida en el presente estudio fue similar a las de las especies de la Unión Americana, con excepción de la TraF, la cual fue mayor para México.

En cuanto a salud, más de la mitad de los géneros arbóreos combinados presentó una Pcv en la categoría pobre, $27.85 \%$, una DenC en la misma categoría, $60.26 \%$, una $\mathrm{TraF}$ severa y $5 \%$ una Mreg de moderada a severa.

Las latifoliadas destacaron por sus pobres Pcv y las coníferas por una severa TraF.

Respecto a los géneros individuales más frecuentes, destacan de bosques templados, Pinus y Quercus y Bursera y Lysiloma, de selvas, todos con TraF en la categoría severa. Bursera también presentó una pobre Pcv y Lysiloma una ligera Mreg. Los estados de Jalisco, Nayarit y Zacatecas presentaron árboles con copas de menor calidad.

Los resultados revelan la importancia de dar seguimiento a este programa de monitoreo iniciado por el INFyS-Conafor para definir a mediano y largo plazo, si los valores de copa obtenidos son "normales". Es indispensable generar umbrales de salud para las especies y condiciones de México, programar y realizar las mediciones de copa en la época apropiada y con personal capacitado. Este es el primer análisis para el indicador condición de copa de bosques y selvas de México.

\section{RECONOCIMIENTOS}

A la Gerencia de Sanidad y al Inventario Nacional Forestal y de Suelos (INFyS) de la Comisión Nacional Forestal (Conafor) por el financiamiento para el análisis de datos y levantamiento de la información de campo, respectivamente. A los líderes del Indicador Condición de Copa del FIA-USDA Forest Service, William A. Bechtold y KaDonna C. Randolph por el apoyo brindado en la capacitación e interpretación de resultados.

\section{REFERENCIAS}

Anderson, R. L., \& Belanger, R. P. (1986). A crown rating method for assessing tree vigor of loblolly and shortleaf pines. En D. R. Phillips (Comp.), Proceedings of the Fourth Biennial Southern Silvicultural Research Conference (pp. 538-543). Atlanta Georgia, Southeaster Forest Experiment Station. Ashville North Carolina.

Bechtold, W. A., \& Randolph, K. C. (2018). FLA Crown Analysis Guide versión 2.0. 42 p. Recuperado de https://www.fs.usda.gov/srsfia/crowns/Crown $\% 20$ Analysis $\% 2$ 0Guide\%20v2.pdf

Bechtold, W. A., Hoffard, W.H., \& Anderson, R.L. (1992). Summary report: forest health monitoring in the South, 1991. USDA For. Serv. Gen. Tech. Rep. SE-81. Recuperado de https://www.srs.fs.usda.gov/pubs/gtr/gtr_se081.pdf

Brandeis, T. J., Escobedo, F. J., Staudhammer, C. L., Nowak, D. J. \& Wayne, C. Z. (2014). San Juan Bay Estuary watershed urban forest inventory (Gen. Tech. Rep. SRS-190). U.S. Department of Agriculture Forest Service, Southern Research Station. Recuperado de https://www.srs.fs.usda.gov/pubs/gtr/gtr_srs190.pdf

Brandeis, T. J., \& Turner, J. A. (2013). Puerto Rico's Forests, 2009. Resour. Bull. SRS-RB-191. Asheville, NC: U.S. Department of Agriculture Forest Service, Southern Research Station. Recuperado de https://www.srs.fs.fed.us/pubs/rb/rb_srs191.pdf

Ferretti, M. (1998). Potential and limitation of visual indices of tree condition. Chemosphere, 36(4-5), 1031-1036. doi: 10.1016/S00456535(97)10167-9

Ferretti, M. (1997). Forest health assessment and monitoring -issues for consideration. Environmental Monitoring and Assessment, 48, 45-72. Recuperado

de https://link.springer.com/content/pdf/10.1023/A:1005748702893.pdf

Hasenauer, H. A., \& Monserud, R. A. (1996). A crown ratio model for Austrian forests. Forest Ecology and Management, 4, 49-60. doi: 10.1016/0378-1127(96)03768-1 
Holdaway, M. R. (1986). Modeling tree crown ratio. The Forestry Chronicle, 62(5), 451-455. doi: 10.5558/tfc62451-5

Inventario Nacional Forestal y de Suelos [INFyS]. (2012). Inventario Nacional Forestaly de Suelos. Informe de Resultados 2004-2009. México: Comisión Nacional Forestal.. Recuperado de https://snigf.cnf.gob.mx/wp-

content/uploads/Resultados $\% 20 \mathrm{Hist} \% \mathrm{C} 3 \%$ B3ricos $\% 20 \mathrm{INFyS} /$ 2004-2009/Informes $\% 20 \mathrm{del} \% 20 \mathrm{INFyS} /$ informe-infys-20042009.pdf

Kenk, G. (1993). Growth in "declining" forests of Baden-Wurttemberg (Southwestern Germany). En R. F., Huettl \& D., MullerDombois (Eds.), Forest decline in the Atlantic and Pacific Region (pp. 202-215). New York: Springe-Verlag.

Krocher, S. D., \& Harris, R. (2007). Tree growth and competition. Forest Stewarship Series 5. ANR Publication 8235. University of California. Recuperado de https://anrcatalog.ucanr.edu/pdf/8235.pdf

Lawrence, R., Moltzan, B., \& Moser, W.K. (2002). Oak decline and the future of Missouri's forests. Missouri Conservationist Magazine, 63(7), 11-18. Recuperado de https://mdc.mo.gov/conmag/2002/060/oak-decline-and-future-missouri-s-forests

Martínez-Vilalta, J., Lloret, F., \& Breshears, D.D. (2012). Droughtinduced forest decline: causes, scope and implications. Biology Letters, 8, 689-691. doi: 10.1098/rsbl.2011.1059

McDowell, N.G., Ryan, M.G., Zeppel, M.J.B., \& Tissue, D.T. (2013). Improving our knowledge of drought-induced forest mortality through experiments, observations, and modeling. New Phytologist 200, 289-293. Recuperado de https://www.fs.fed.us/rm/pubs_other/rmrs_2013_mcdowell_n 002.pdf

Michel, A., \& Seidling W. (Eds.) (2017). Forest condition in Europe: 2017 Technical Report of ICP Forests. Report under the UNECE Convention on Long-Range Transboundary Air Pollution (CLRTAP). BFW-Dokumentation. 24/2017. Vienna: BFW Austrian Research Centre for Forests. Recuperado de https://www.mapa.gob.es/es/desarrollo-rural/temas/politicaforestal/informetecnico2017ingles_tcm30-441604.pdf

Millers, I., Shriner, D., \& Rizzo, D. (1989). History of hardwood decline in the Eastern United States (Gen. Tech. Rep. NE-126). Radnor, PA: U.S. Department of Agriculture Forest Service, Northeastern Forest Experiment Station. Recuperado de https://www.fs.fed.us/ne/newtown_square/publications/techn ical_reports/pdfs/scanned/gtr126.pdf

Morin, R.S., Randolph, K.C., \& Steinman, J. (2015). Mortality rates associated with Crown health for Eastern forest tree species. Environmental Monitoring and Assessment, 187(3), 87-98. Recuperado de https://link.springer.com/content/pdf/10.1007\%2Fs10661015-4332-x.pdf

Morin, R. S., Steinman, J., \& Randolph, K. C. (2012). Utility of tree crown condition indicators to predict tree survival using remeasured forest inventory and analysis data. Moving from Status to Trends: Forest Inventory and Analysis Symposium 2012 (Gen. Tech. Rep. NRS-P-105). Recuperado de https://www.nrs.fs.fed.us/pubs/gtr/gtr-nrs-p105papers/33morin-p-105.pdf

Oak, S. W., Steinman, J. R., Starkey, D. A., \& Yockey, E. K. (2004). Assessing oak decline incidence and distribution in the Southern U.S. using Forest Inventory and analysis data (Gen. Tech. Rep. SRS-73). Asheville, NC: U.S. Department of Agriculture, Forest Service, Southern Research Station. Recuperado de https://www.srs.fs.usda.gov/pubs/gtr/gtr_srs073/gtr_srs073oak001.pdf

Ochoa, M. (2001). Información y análisis para el manejo forestal sostenible: integrando esfuerzos nacionales e internacionales en 13 paises tropicales en América Latina (Proyecto GCP/RLA/133/EC). Proceso de Montreal. Países: Argentina, Chile, México y Uruguay. Santiago de Chile. Recuperado de http://www.fao.org/tempref/GI/Reserved/FTP_FaoRlc/old/ proyecto/rla133ec/CI\%20-\%20pdf/Proceso\%20Montreal.PDF

Oswalt, C. M., Oswalt, S. N., Johnson, T. G., Chamberlain, J. L., Randolph, K.C., \& Coulston, J.W. (2009). Tennessee's forests, 2004. Resource Bulletin SRS-144. Asheville, NC: U.S. Department of Agriculture Forest Service, Southern Research Station. Recuperado de https://www.srs.fs.usda.gov/pubs/32506

Pontius, J., \& Hallet, R. (2014). Comprehensive methods for earlier detection and monitoring of forest decline. Forest Science, 60(6), 1156-1163. Recuperado de https://www.fs.fed.us/nrs/pubs/jrnl/2014/nrs_2014_pontius_ 001.pdf

Randolph, K. C., Morin, R. S., \& Steinman, J. (2010a). Descriptive statistics of tree crown condition in the Northeastern United States (Gen. Tech. Rep. SRS-124). Asheville, NC: U.S. Department of Agriculture Forest Service, Southern Research Station. Recuperado de https://www.nrs.fs.fed.us/pubs/35855

Randolph, K. C., Morin, R. S., \& Steinman, J. (2010b). Descriptive statistics of tree crown condition in the North Central United States (Gen. Tech. Rep. SRS-125). Asheville, NC: U.S. Department of Agriculture Forest Service, Southern Research Station. Recuperado de https://www.srs.fs.usda.gov/pubs/gtr/gtr_srs125.pdf

Randolph, K. C., Campbell, S. J., \& Christensen, G. (2010). Descriptive statistics of tree crown condition in California, Oregon, and Washington (Gen. Tech. Rep. SRS-126). Asheville, NC: U.S. Department of 
Agriculture Forest Service, Southern Research Station. Recuperado de https://www.srs.fs.usda.gov/pubs/gtr/gtr_srs126.pdf

Randolph, K. C., \& Thompson, M. T. (2010). Descriptive statistics of tree crown condition in the United States Interior West (Gen. Tech. Rep. SRS-127). Asheville, NC: U.S. Department of Agriculture Forest Service, Southern Research Station. Recuperado de https://www.srs.fs.usda.gov/pubs/gtr/gtr_srs127.pdf

Randolph, K.C. (2009). Crown condition. En M. J., Ambrose \& B. L. Conkling (Eds.), Forest health monitoring: 2006 national technical report (pp. 65-110) (Gen. Tech. Rep. SRS-117). Asheville, NC: U.S. Department of Agriculture Forest Service, Southern Research Station. Recuperado de https://www.fs.usda.gov/treesearch/pubs/34402

Randolph, K.C. (2006). Descriptive statistics of tree crown condition in the southern United States and impacts on data analysis and interpretation (Gen. Tech. Rep. SRS-94). Ashville, NC: U.S. Department of Agriculture Forest Service, Southern Research Station.

Randolph, K. C. (2004). Inter- and intra-species variation in three crown condition indicators for seven tree species in theSoutheastern United States. Ph.D. Dissertation, University of Tennessee. Recuperado de https://trace.tennessee.edu/utk_graddiss/2213

Randolph, K.C., \& Moser, J. W. (2004). An evaluation of change in tree crown characteristics to assess forest health in two Indiana State Parks. Northern Journal of Applied Forestry 21(1), 50-55. Recuperado de http://agris.fao.org/agris-search/search.do?recordID=US201301015157

Riitters, K., \& Tkacz, B. (2004). The U.S. forest health monitoring program. En B. Wiersma (Ed.), Environmental Monitoring (pp. 669683). Boca Raton, FL: CRC Press.

Saavedra-Romero, L. L., Alvarado-Rosales, D., Hernández-de la Rosa, P., Martínez-Trinidad, T., Mora-Aguilera, G., \& Villa-Castillo, J. (2016). Condición de copa, indicadores de salud en árboles urbanos del Bosque San Juan de Aragón, Ciudad de México. Madera y Bosques, 22(2), 15-27. doi: 10.21829/myb.2016.2221321

Schomaker, M. E., Zarnoch, S. J., Bechtold, W. A., Latelle, D. J., Burkman, W. G., \& Cox, S. M. (2007). Crown condition classification: a guide to data collection and analysis (Gen, Tech. Rep. SRS-102). Ashville, NC: U.S. Department of Agriculture, Forest Service, Southern Research Station. Recuperado de https://www.srs.fs.usda.gov/pubs/27730

Sharma, R. P., Vacek Z, Vacek S, Podrázský V. \& Jansa V. (2017). Modelling individual tree height to crown base of Norway spruce (Picea abies (L.) Karst.) and European beech (Fagus sylvatica L.). PLOS ONE, 12(10), e0186394. doi: 10.1371/journal.pone.0186394
Steinman, J. (2000). Tracking the health of trees over time on forest health monitoring plots. En M. Hansen \& T. Burk (Eds.). Integrated tools for natural resources inventories in the 21st century (pp. 334-339) (Gen. Tech. Rep. NC-212). U.S. Department of Agriculture Forest Service, North Central Forest Experiment Station. Recuperado de https://www.fs.usda.gov/treesearch/pubs/2271

The Montréal Process. (2015). Criteria and indicators for the conservation and sustainable management of temperate and boreal forests (5a ed.). Recuperado de https://www.montrealprocess.org/documents/publications/tec hreports/MontrealProcessSeptember2015.pdf

Tkacz, B., Moody, B., Villa-Castillo, J., \& Fenn, M. E. (2008). Forest health conditions in North America. Environmental Pollution, 155, 409-425. Recuperado de https://www.fs.usda.gov/pnw/publications/forest-healthconditions-north-america

United States Department of Agriculture [USDA]. (2005). Crown: measurements and sampling. In Forest Inventory and Analysis National core field guide: field data collection procedures for phase 3 plots. Version 3.0. Section 12, 1-21. Recuperado de https://www.fia.fs.fed.us/library/field-guides-methodsproc/docs/2006/p3_3-0_sec12_10_2005.pdf

United States Department of Agriculture [USDA]. (2004). National report on sustainable forest - 2003. United States Department of Agriculture. FS-766. 139 p. Recuperado de https://www.fs.fed.us/research/sustain/docs/nationalreports/2003/2003-sustainability-report.pdf

Westfall, J. A., Westfall, M. B., \& Randolph. K. C. (2019). Modelling relations between compacted and uncompacted crown ratio for the Northern United States. Forest Science, 65(5), 593-601. doi: 10.1093/forsci/fxz029

Widmann, R. H., Cook, G. W., Barnett, C. J., Butler, B. J., Griffith, D. M., Hatfield, M. A., Kurtz, C. M., Morin, R. S., Moser, W. K., Perry, C. H., Piva, R. J., Riemann, R., \& Woodall, C.W. (2012). West Virginia's Forests 2008. Resourse Bulletin NRS-61. Newtown Square, PA: U.S. Department of Agriculture, Forest Service, Northern Research Station. Recuperado de https://www.fs.fed.us/nrs/pubs/rb/rb_nrs61.pdf

Zarnoch, S. J., Bechtold, W. A., \& Stolte, K. W. (2004). Using Crown condition variables as indicators Forest health. Canadian Journal Forest Research, 34, 1057-1070. Recuperado de https://www.srs.fs.usda.gov/pubs/ja/ja_zarnoch001.pdf 
Manuscrito recibido el 07 de febrero de 2020

Aceptado el 25 de mayo de 2020

Publicado el 21-de abril de 2021

Este documento se debe citar como:

Alvarado-Rosales, D., Saavedra-Romero, L. L., Franco-Islas, Y., Villa-Castillo, J., \& y Quiroz-Reygadas, D. A. (2021). Condición de copa de bosques y selvas de México: Análisis 2014. Madera y Bosques, 27(1), e2712114. doi: 10.21829/myb.2021.2712114

Madera y Bosques por Instituto de Ecología, A.C. se distribuye bajo una Licencia Creative Commons Atribución-NoComercialCompartirlgual 4.0 Internacional. 